\title{
Redes sociales y promoción de destinos turísticos termales de la Eurorregión Galicia-Norte de Portugal
}

\section{Social networks and promotion of thermal tourism destinations of the Euro-region Galicia-Northern Portugal}

\author{
Eva Sanchez Amboage*, Valentín Alejandro Martínez Fernández*, Oscar Juanatey Boga*, \\ María Magdalena Rodríguez Fernández* \\ * Universidad de A Coruña, España
}

Resumen

\begin{abstract}
La eurorregión Galicia-Norte de Portugal conforma el territorio con más recursos termales de Europa, lo cual ha posibilitado la construcción de un elevado número de establecimientos termales, referentes en su sector. Ante el escenario de constante cambio y evolución que ha experimentado el Turismo Termal, las Tecnologías de la Información y Comunicación (TICS) han sido relevantes para optimizar y mejorar la gestión y comercialización de su actividad turística. La presente investigación analiza la importancia de las redes sociales como medio de promoción del turismo termal a través de la opinión de los responsables de los establecimientos termales de Galicia y del Norte de Portugal. Las entrevistas estructuradas a los gestores de los balnearios indicados ha sido la técnica empleada para extraer la información necesaria, la cual se ha apoyado en el uso de la herramienta de análisis cualitativo y cuantitativo MAXQDA 2011. Los resultados evidencian que, si bien la mayoría de los responsables de los balnearios entrevistados coinciden en destacar la importancia de las redes sociales en la promoción online de sus respectivos establecimientos, se aprecia un desinterés generalizado en lo concerniente a su utilización.
\end{abstract}

Palabras-clave: Turismo Termal; Eurorregión Galicia-Norte de Portugal; Redes Sociales; MAXQDA.

Abstract

The euro-region Galicia-Northern Portugal is the territory with more thermal resources in Europe, which has made possible the construction of a high number of thermal establishments, benchmarks in their sector. Against an ever-changing and evolving scenario in thermal tourism, Information and Communication Technologies (ICT) have been relevant to improve the management and commercialization of its tourism activity. The present research assesses the relevance of social media as platforms for promoting thermal tourism hanks to the opinion of people responsible for thermal establishments in Galicia and Northern Portugal. The method used was the structured interview to health resorts managers, supported by the use of the tool MAXQDA 2011 for qualitative and quantitative analysis. Results show that, while most of the health resorts managers highlight the relevance of social media in the online promotion of their establishments, there is a general indifference as regards the use of these platforms.

Keywords: Thermal Tourism; Euro-region Galicia-Northern Portugal; Social Networks,;MAXQDA

Submitted: 13th December 2016

Accepted: 27th November 2018

\section{Introducción}

En el sector turístico de Galicia y del Norte de Portugal, el termalismo constituye una actividad clave para el desarrollo social, económico y medioambiental del lugar. La eurorregión es uno de los espacios de la

Copyright (c) 2019 (Eva Sanchez Amboage, Valentín Alejandro Martínez Fernández, Oscar Juanatey Boga, María Magdalena Rodríguez Fernández). Licensed under the Creative Commons Attribution-NonCommercial Generic (cc by-nc). Available at http://obs.obercom.pt. 
Península Ibérica con mayor riqueza de aguas mineromedicinales, en cuanto a calidad y número de manantiales se refiere. Por eso, el Turismo Termal se presenta como un modelo turístico atractivo, capaz de generar empleo y atraer a turistas de diferentes lugares de España, de Portugal y del resto de Europa. De acuerdo a la argumentación de Gonçalves, Fraiz y Manosso (2013), el uso de Internet como estrategia para fortalecer la marca en el sector turístico tiene tres beneficios: 1) Económico, a través de las promociones realizadas en la página y del aumento de la demanda de un determinado producto; 2) Informativo, al contactar directamente con el cliente y recoger sus opiniones; 3) Branding, al contribuir a posicionar la marca, haciéndola más atractiva para el cliente.

Dichos beneficios pueden ser perfectamente aplicables al sector turístico termal de Galicia y Norte de Portugal, el cual, en los últimos años no sólo ha desarrollado y mejorado sus servicios termales y sus infraestructuras, sino que también ha progresado en su promoción y comercialización a través de Internet y más concretamente de las redes sociales ${ }^{1}$.

En este sentido, puede decirse que los establecimientos termales emplean distintas técnicas de promoción, las cuales se dividen en dos grandes grupos: a) Las tradicionales, aplicadas desde hace años (comunicación a través de asociaciones, asistencia a ferias, publicity, artículos en revistas, folletos, "boca-oreja", pagina web, etc.) y b) Las más actuales, entre las cuales se encuentran las redes sociales. En este último caso, fundamentalmente por el número de balnearios que las utilizan, cabe reseñar que Facebook y Twitter destacan como redes sociales virtuales generalistas y Booking y TripAdvisor como redes sociales virtuales especializadas ${ }^{2}$ en el sector turístico.

El hecho de posibilitar el contacto de manera instantánea con un perfil de personas interesadas y predispuestas a recibir diariamente información turística en forma de comentarios, fotografías, vídeos, etc., convierte a las redes sociales en herramientas eficientes para este tipo de promoción. Pero, llegados a este punto, cabe plantear las siguientes cuestiones: ¿Cuál es la opinión real de los responsables de los balnearios sobre este nuevo canal online para dar a conocer su establecimiento? ¿Están dispuestos a invertir recursos en este medio? ¿Cómo organizan su trabajo en la empresa con la llegada de las redes sociales?

Conforme a las preguntas anteriores, se propone el siguiente objetivo general: Estudiar y analizar la opinión de los responsables de los establecimientos termales de Galicia y del Norte de Portugal, sobre las redes sociales como medio de promoción turística termal.

Las entrevistas estructuradas a los responsables de los balnearios de la eurorregión, ha sido la técnica empleada para extraer la información necesaria para esta investigación; para ello, se ha utilizado como

\footnotetext{
${ }^{1}$ En este artículo los términos: redes sociales, social media y medios sociales son empleados como sinónimos. Es necesario aclarar que Cavalganti y Sobejano (2011) hablan de medios sociales, en lugar redes sociales, término más extendido en España. Se intenta establecer una distinción entre las redes sociales virtuales (medios sociales) que han permitido la interacción entre usuarios de forma virtual y las redes sociales reales (aquellos grupos de personas físicas que se unen por un interés común) (Celaya, 2008).

2 Dentro de las redes sociales virtuales, Celaya (2008) diferencia entre "redes sociales generalistas" (ej. Facebook y Twitter), "redes sociales profesionales" (Ej. LinkedIn) y "redes sociales especializadas" (ej. En diferentes sectores, ámbitos, etc.) Para el caso que nos ocupa, y siguiendo esta última clasificación de redes sociales especializadas en el sector turístico se podrían citar ejemplos como: MiNube, TripAdvisor y Booking. Cabe señalar al respecto que Booking y TripAdvisor pueden contemplarse, al igual que indica Hernández, Fuentes y Morini (2012), como proveedores de información hotelera en internet, en lugar de redes sociales. Sin embargo, si atendemos a cualquier definición de red social, como pueden ser las efectuadas por Christakis y Flowler (2010), Kaplan y Haenlein (2010), Ellison, Steinfield y Lampe (2007) o Scott (2010) los Medios Sociales providencian el medio por el que las personas generan ideas, contenidos, pensamientos y relaciones online; compartiendo por tanto las característica de páginas como Booking y TripAdvisor.
} 
herramienta de análisis cualitativo y cuantitativo MAXQDA 2011, la cual ha facilitado la generación de significado y las conclusiones del estudio.

El presente trabajo se estructura en tres bloques: a) Teórico, compuesto por la información sobre turismo termal y redes sociales, b) Metodológico, donde se explica el método utilizado en la investigación y finalmente c) Empírico, a través de la presentación de los resultados y las conclusiones del estudio.

\section{Eurorregión termal Galicia-Norte de Portugal}

Después de años de constante evolución, el Turismo Termal se ha convertido en una actividad capaz de captar a usuarios de todas las edades, atraídos por motivos de carácter médico, de ocio o de relax que buscan disfrutar de una experiencia, donde el agua mineromedicinal es el elemento fundamental.

En lo que concierne a su definición, el Turismo Termal se enmarca dentro del Turismo de Salud que, según el Ministerio de Industria Energía y Turismo (2013) ${ }^{3}$ y Goodrich y Goodrich (1987), consiste en el proceso por el cual una persona viaja para recibir servicios de salud en un país diferente a aquel en el que reside. El motivo del viaje es, en sentido amplio, la búsqueda de estos servicios de salud. Del mismo modo, el Governo de Portugal (2014) ${ }^{4}$, considera que el Turismo de Salud es el fenómeno caracterizado por el desplazamiento de turistas cuya motivación principal es la obtención de beneficios relacionados con la salud. Entre sus propósitos se encuentra el hecho de articular el valor médico con el turístico, los cuales están directa e indirectamente asociados.

El desarrollo del Turismo de Salud, explica Hoz (2013), ha sido diferente en cada continente, cuyas desigualdades se han forjado a causa de creencias, conocimientos, tradiciones y culturas de cada país o región. En Europa, la práctica del Turismo de Salud ha girado en torno a los establecimientos termales y tratamientos con agua de mar, principalmente por el amplio número de fuentes termales, minerales y de costas.

En el caso concreto de la Península Ibérica, Galicia y el Norte de Portugal eran las regiones que, debido a la calidad de sus aguas, concentraban un mayor número de "Ciudades Aquae"; es decir, enclaves de los que manaban aguas mineromedicinales. Así, las ciudades reconocidas por aquel entonces, según Casal y González (2010) eran: Aquae Celenae (Caldas de Reis), Aquae Querquennae (Baños de Bande), Aquae Originis (Riocaldo, Lobios) y Aquae Flavianae (Chaves, Portugal).

Desde esta edad de oro del termalismo en la época romana, los balnearios han pasado por distintas etapas, algunas como la Edad Media, donde se enfrentaban a un "período de hibernación", u otras como la Ilustración, en la cual se enmarca el resurgir de la hidroterapia. Es en el siglo XVIII, con la Ilustración, cuando, según Casal y González (2010), cobran gran importancia en los balnearios prácticas como: los juegos de azar, el golf, los casinos, los eventos culturales, etc. con el claro objetivo de proporcionar un mayor entretenimiento a las clases pudientes. De esta forma, los establecimientos termales se complementan con hoteles de lujo, naturaleza, monumentos, fuentes y un largo etc. Se habla entonces del

\footnotetext{
${ }^{3} \mathrm{http}: / /$ goo.ql/trs]mc

${ }^{4}$ http://goo.gl/MPLQyg
} 
arranque definitivo del termalismo en España y Portugal, así como en el resto de Europa, que se verá acentuado en el siglo XIX y XX.

Es por tanto a partir de 1990 cuando los balnearios se reinventan, al ofrecer no sólo un lugar donde tratar dolencias, sino también un espacio de culto al cuerpo y de relajación. A todo esto, tal y como apuntan Bonfada, Bonfada, Gândara y Brea (2008), deben de sumarse las importantes renovaciones e inversiones realizadas por los establecimientos en los últimos años, sobre todo en acondicionamiento, tecnología y promoción.

El ámbito geográfico donde se sitúa esta investigación se corresponde con la región fronteriza "GaliciaRegião Norte de Portugal", también conocida como "eurorregión Galicia-Norte de Portugal" o "Región GaliciaNorte de Portugal". Dicho territorio se ubica al Noreste de la Península Ibérica y comprende la Comunidad Autónoma de Galicia (A Coruña, Lugo, Ourense y Pontevedra) así como la Región del Norte de Portugal (Minho-Lima, Cávado, Ave, Tâmega, G.Porto, Entre Douro e Vouga, Alto Tras-os-Montes, Douro).

\section{Las redes sociales en la industria turística}

Las nuevas tecnologías originaron una nueva forma de percibir el mundo y de comunicación entre la sociedad en general (Amaral, 2008). Según Martínez (2011a) las TICs han propiciado que el turista actual se convierta en un cliente experimentado y planificador, capaz de buscar información, fundamentalmente, a través de Internet, medio que se ha convertido en la mayor fuente de información para viajeros y en una plataforma para el negocio turístico (Pan, MacLaurin y Crotts, 2007).

Del mismo modo, Álvarez, Benamou, Fernández y Solé $(2011)^{5}$ defienden la existencia de una transformación clara de los sectores debido a Internet, entre los cuales se encuentra el sector turístico. En su estudio los autores muestran que el gran desarrollo de la actividad turística se debe principalmente a tres características: Intensidad de uso de información en la planificación del viaje; comercialización de un servicio y no de bienes físicos; y, finalmente, la rápida adaptación del sector al canal online.

En lo referente a la promoción turística, sin olvidar tampoco las nuevas tecnologías, Cruz (2005) explica que entre los objetivos que persigue este tipo de promoción se encuentran: informar, persuadir, inducir, recordar, comunicar y sensibilizar a los clientes actuales y potenciales; así como, atraer y conquistar la fidelidad de los consumidores. Con el fin de alcanzar estos propósitos es necesario que la promoción turística esté alineada con la planificación de marketing para obtener los resultados esperados por los gestores o empresas del ámbito turístico. De este modo, a juicio de Martínez (2012), la promoción turística supone comunicación, transmisión de información de los organismos o empresas responsables a los potenciales turistas, susceptible de ser realizada a través de distintos medios. Entre estos medios se encuentran las redes sociales, uno de los canales más actuales y novedosos para el desarrollo de la promoción turística, en general, y del sector turístico termal, en particular. En este sentido, Hudson y Thal (2013), Kang y Schuett (2013), Munar y Steen (2014) o Allfacebook (2013) ${ }^{6}$, consideran que los medios sociales han cambiado fundamentalmente el proceso de decisión de compra del consumidor y en la última década ha surgido una

\footnotetext{
${ }^{5}$ https://goo.gl/At8h3r

${ }^{6}$ https://goo.gl/gmfx04
} 
visión más sofisticada en la evaluación de un producto y en la toma de decisiones, donde los usuarios del social media son los protagonistas (García, 2010).

En el ámbito turístico, las comunidades virtuales representan una gran oportunidad para los viajeros, pues en ellas es posible encontrar las opiniones sobre destinos turísticos potenciales, los servicios de los que se puede disfrutar (hoteles, restaurantes, etc.) y, sobre todo, recibir de primera mano, sin intermediarios comerciales, información respecto a las experiencias vividas por otras personas en el destino turístico al que el viajero piensa acudir. (Casaló, Flavián, Guinalín, 2009)

Ante este contexto de cambio, evolución y progreso en el que se encuentra el sector turístico y el termalismo, las Tecnologías de la Información y Comunicación (TICs) desempeñan un papel cada vez más relevante en aras de optimizar y mejorar la gestión y comercialización de su actividad.

\section{Metodología}

Al centrarse esta investigación en un estudio individual de la situación de los balnearios de Galicia y del Norte de Portugal en el año 2015, se considera que el método con mejor adaptación a este trabajo es el denominado como estudio de casos.

El estudio de casos, según Martínez (2011b) es una técnica muy cuestionada al considerar, distintos investigadores, como Yin (1993), que sólo puede utilizarse para estudios exploratorios y tiene un papel bastante limitado en la investigación, con un diseño preexperimental. Del mismo modo, Coller (2000) también apunta como uno de los errores de muchos sociólogos el hecho de creer que el método del caso es sólo apropiado para un tipo de investigación exploratoria, de carácter cualitativo, generadora de conocimientos primarios útiles pero irrelevantes, al no ser posible extrapolarlos a una población o hacer generalizaciones a partir de uno, o de unos pocos casos.

Aun cuando los investigadores de corte cuantitativo cuestionan la investigación cualitativa, al alegar que sus conclusiones no son generalizables estadísticamente, Castro (2010) considera que, específicamente con el estudio de casos, no se representa a una muestra de una población o universo, pero son cuestiones específicas las que se estudian buscando con esta metodología una generalización analítica y no estadística. Asimismo, Coller (2000), Chetty (1996) y Yin (1994) afirman que es una herramienta valiosa, entre otras disciplinas, para las Ciencias Sociales. Esto es también defendido por De la Cruz Pulido y Pulido (2014), al explicar que, a pesar de las críticas a este método, éste es cada vez más utilizado, también en investigaciones de carácter turístico.

Autores como Villareal y Landeta (2007) afirman que los inconvenientes del estudio de casos pueden eliminarse y convertirse en un método científico válido si se sigue un diseño metodológico riguroso, el cual vele por el alcance de niveles de validez y confiabilidad más altos.

El método del caso puede emplearse, por tanto, como una herramienta de exploración, pero también de comprobación y construcción de teorías. Además, en la mayoría de las ocasiones, Coller (2000) destaca la bondad de este estudio al permitir aproximarse a los actores de manera que se pueda realizar la comprensión e interpretación de sus acciones con éxito.

Cabe precisar al respecto, que esta investigación se centra en el estudio de casos como estrategia metodológica, compuesto por dos estudios individuales; es decir, por unidades de análisis, que comparten 
características comunes y, tras ser examinados de forma individual, se construyen ciertas caracterizaciones más generales.

Se consideran así los siguientes: Caso A: los balnearios de Galicia y Caso B: los balnearios del Norte de Portugal y que, a su vez, están integrados por los establecimientos termales en funcionamiento, en el año 2015, de cada región.

Ante esta situación, la entrevista estructurada será empleada como técnica de recogida de datos. Según Bell (2004), esta herramienta permite agregar y cuantificar los resultados de forma sencilla. Puede adoptar la forma de un cuestionario o de una lista que sea completada por el entrevistador y no por el entrevistado, ahorra tiempo a la hora de analizar los resultados y tiene la garantía de que todos los temas son tratados. Ha de matizarse que, a lo largo de esta investigación y en la realización de las entrevistas, se han encontrado algunas dificultades para conseguir la participación de todos los responsables de los establecimientos, debido principalmente al desinterés en contestar a la entrevista. A pesar de ello, se han obtenido, conforme se puede observar en la Tabla 1, un total de 24 entrevistas (12 de balnearios de Galicia y 12 de balnearios del Norte de Portugal).

Tabla 1: Balnearios de la eurorregión Galicia-Norte de Portugal en funcionamiento en el año $2015^{7}$

\begin{tabular}{ll}
\hline Caso A: Balnearios de Galicia 2015 & $\begin{array}{l}\text { Caso B: Balnearios del } \\
\text { Norte de Portugal } 2015\end{array}$ \\
\hline 1. Balneario de Carballo* & $\begin{array}{l}\text { 1. Termas de Monção* } \\
\text { 2. Balneario de Compostela* }\end{array}$ \\
3. Balneario de Guitiriz* & 3. Termas de Caldelas \\
4. Balneario Baños da Brea* & 4. Termas de Moimenta* \\
5. Balneario Río Pambre* & 5. Termas do Gerês \\
6. Balneario de Lugo* & 6. Termas Carvalhelhos \\
7. Balneario Oca Aguas Santas & 7. Termas de Chaves* \\
8. Balneario Baños de Molgas & 8. Vidago Palace* \\
9. Balneario de Lobios Caldaria* & 9. Pedras Salgadas Spa \& Nature Park* \\
10. Balneario Arnoia Caldaria* & 10. Caldas de Carlão \\
11. Balneario Laias Caldaria* & 11. Termas de São Lourenço* \\
12. Balneario Davila & 12. Termas da Terronha* \\
13. Balneario de Cuntis & 13. Termas das Taipas* \\
14. Balneario de Acuña & 14. Termas das Caldas de Vizela* \\
15. Gran Hotel La Toja & 15. Termas das Caldas da Saúde* \\
16. Balneario Isla de La Toja* & 16. Termas de Entre os Ríos \\
17. Balneario Caldelas de Tui & 17. Termas de SãoVicente \\
18. Balneario de Mondariz* & 18. Caldas de Arêgos* \\
19. Balneario de O Carballiño* & 19. Termas de São Jorge* \\
\hline
\end{tabular}

Fuente: Elaboración propia a partir del Observatorio Nacional de Termalismo y Desarrollo Rural (2015) ${ }^{8}$ y la Entidad Regional de Turismo Porto e Norte ${ }^{9}$

\footnotetext{
${ }^{7}$ Los balnearios señalados con asterisco $(*)$ son los que han accedido a ser entrevistados.

${ }^{8} \mathrm{http}: / /$ goo.gl/ibk7bO

9 http://www.portoenorte.pt/
} 
Tal y como se aprecia en la tabla anterior, de los 19 balnearios gallegos en funcionamiento en el año 2015, han aceptado la entrevista 12, los cuales están representados por 10 balnearios, debido a que el grupo "Caldaria Balnearios" está integrado por 3 establecimientos termales de la provincia de Ourense. Por este motivo, al poseer un único departamento de comunicación para los 3 establecimientos, solamente se ha realizado una entrevista. Por consiguiente, de las diez personas finalmente entrevistadas, seis eran mujeres y cuatro hombres, con funciones que van desde directores, propietarios y responsables de balnearios hasta jefes del departamento de recepción, comercialización y comunicación.

Respecto a los balnearios del Norte de Portugal, de los 19 que durante el año 2015 se encontraban en funcionamiento, 12 son los que han accedido a ser entrevistados. La muestra de establecimientos a analizar se equipara, por tanto, al caso gallego. Al estudiar ahora más detalladamente el perfil de los entrevistados cabe precisar que de las 12 personas, 8 eran mujeres y 4 hombres, las cuales desempeñaban funciones que van desde directores y propietarios del balneario, hasta responsables de departamento de comunicación y marketing.

Una vez obtenidos los datos de las entrevistas, se procedió al estudio y presentación de los resultados con el objetivo de extraer las conclusiones pertinentes de la investigación. Según Miles, Huberman y Saldaña (2013) existen diferentes formas de realizar informes de resultados y presentar adecuadamente un estudio. Para esta investigación la herramienta de análisis cualitativo y cuantitativo MAXQDA 2011 ha facilitado la generación de significado a través del sistema de codificación, al igual que verifican autores como: Galán, Edwards, Nelson y Van del Wal (2015), Gibbs (2013) ${ }^{10}$, Booth (2014) Coryn, Schröter y McCowen (2014) en diversas áreas de estudio.

En el ámbito de la investigación cualitativa los códigos son indicadores, etiquetas, categorías, conceptos, que permiten asignar unidades de significado a la información descriptiva o inferencial compilada durante una investigación. En otras palabras, son recursos mnemónicos utilizados para identificar o marcar los temas específicos en un texto. (Fernández, 2006).

De este modo, se ha codificado la información obtenida de las entrevistas estructuradas, para así poder agruparlas en patrones recurrentes y temas emergentes en el análisis. La codificación, de acuerdo a como explica Patton (2002), fuerza al investigador a hacer juicios acerca del significado de bloques contiguos de texto y permite eliminar el caos y la confusión que habría sin algún sistema de clasificación. Esto implica un trabajo intelectual y mecánico que permite codificar los datos, encontrar patrones, etiquetar los temas y desarrollar sistemas de categorías, lo cual significa analizar el contenido central de las entrevistas estructuradas para determinar qué es representativo, y a partir de ahí, reconocer patrones y transformarlos en categorías significativas y temas. En este caso, las categorías y subcategorías empleadas son las siguientes:

\footnotetext{
${ }^{10}$ https://goo.gl/QBrFKz
} 
Tabla 2: Categorías y subcategorías de la investigación

\begin{tabular}{|c|c|}
\hline Categorías & Subcategorías \\
\hline \multirow{3}{*}{$\begin{array}{l}\text { 1.Entendiendo las redes } \\
\text { sociales }\end{array}$} & Definición de red social. \\
\hline & Importancia de las redes sociales para el establecimiento. \\
\hline & Ventajas y desventajas de las redes sociales. \\
\hline \multirow[t]{2}{*}{$\begin{array}{l}\text { 2. ¿Qué persiguen en las } \\
\text { redes sociales? }\end{array}$} & $\begin{array}{l}\text { Objetivo que persiguen con su presencia en las redes } \\
\text { sociales. }\end{array}$ \\
\hline & Perfil de usuario que buscan captar en este medio. \\
\hline \multirow{5}{*}{$\begin{array}{l}\text { 3.Actividad en las redes } \\
\text { sociales }\end{array}$} & Comentarios recibidos en las redes sociales. \\
\hline & Contenido más apropiado para ser publicado. \\
\hline & La figura del Community Manager en los establecimientos \\
\hline & termales. \\
\hline & Redes sociales que mejor se adaptan a los balnearios. \\
\hline 4.Percepción de rentabilidad & Rentabilidad de las redes sociales para un balneario. \\
\hline $\begin{array}{l}\text { 5.Autoevaluación } \\
\text { previsión futura }\end{array}$ & $\begin{array}{l}\text { Gestión adecuada o inadecuada de los medios sociales. } \\
\text { Previsión de futuro de las redes sociales. }\end{array}$ \\
\hline
\end{tabular}

Fuente: Elaboración propia

Así, para esta investigación, se han utilizado los estándares de calidad de Miles, Huberman y Saldaña (2013), al ofrecer cada uno de ellos distintos consejos que ayudan a perfeccionar el trabajo a través de la objetividad, confiabilidad, credibilidad, transferibilidad y la utilización.

\section{Resultados}

Los resultados de la investigación se presentan de acuerdo a las categorías realizadas para estructurar la información en el programa MAXQDA2011.

\section{Entendiendo las redes sociales}

En primer lugar y ante las definiciones recabadas, se observa que la comunicación entre empresa-cliente, es la percepción principal de las personas encargadas de los establecimientos termales sobre las redes sociales, tanto de Galicia como del Norte de Portugal. Además, una de las ideas principales extraídas de las definiciones de los entrevistados es el concepto del social media como herramienta de "comunicación futura" entre las empresas termales y sus clientes, al indicar que se trata de una herramienta para comunicarse tanto con sus clientes actuales como potenciales. Por un lado, identifican al "joven de hoy como el sénior de mañana", siendo este "joven" quien más utiliza actualmente las redes sociales. Por eso, consideran necesario contactar con este usuario debido a que en el futuro podrá convertirse en un cliente termal clásico. Por otra parte, los responsables de los balnearios están muy interesados en concienciar a las personas, principalmente jóvenes, sobre la importancia de la prevención en materia de termalismo. El hecho de 
emplear las aguas mineromedicinales de forma habitual incide directamente en la prevención de determinadas enfermedades y dolencias, algo de lo cual se podrán beneficiar con el paso del tiempo.

En lo referente a las palabras que definen a los medios sociales se descubre que, a excepción de los términos "comunicación" y "promoción", vocablos repetidos en varias ocasiones, el resto de establecimientos de Galicia mencionan otros conceptos diferentes. Sin embargo, entre los balnearios del Norte de Portugal, la palabra "comunicación"está más extendida.

También se recogen términos que contienen un mayor significado como son: "futuro" y "necesarias" para los balnearios gallegos y "oportunidad", "futuro", "fundamental", "actualidad" y "presencia" para los casos del Norte de Portugal, aludiendo quizás a la ocasión que presentan este tipo de medios en materia de "promoción" y "publicidad". Se destaca que, en general, existe la tendencia de asociar los Medios Sociales e incluso Internet, con modernidad y futuro, añadiendo nuevamente significado al concepto de "comunicación futura".

En cuanto a la importancia atribuida por los responsables de los establecimientos a las redes sociales se averigua que dos balnearios de Galicia y uno del Norte de Portugal, entienden que la presencia en los Medios Sociales no es imprescindible para un balneario. Entre las aportaciones destaca la explicación del Hotel Balneario de Carballo: "Para nuestro tipo de cliente las redes sociales no tienen demasiada importancia".

La justificación de este establecimiento coincide con una opinión generalizada de quienes asocian los servicios balnearios fundamentalmente a personas mayores, donde se integra la actividad balnearia en la vertiente más puramente médica del Turismo de Salud. De las palabras del balneario de Carballo se vislumbra, además, que las personas mayores no son usuarias activas de los medios sociales y, por lo tanto, no es un medio que les preocupe.

A lo anterior podría añadirse la explicación de Hyde (2007) o Zins (2009), los cuales argumentan que la mayor parte del proceso de búsqueda de información turística todavía no se centra en los sitios de redes sociales, si bien el uso de Internet como fuente de información para el viaje esté extendido. Sin embargo, el resto de balnearios coincide en que el social media sí es importante y relevante para un establecimiento termal, pensamiento defendido incluso por aquellos balnearios sin presencia, por el momento, en ninguna red social, como es el caso de: Termas de São Lourenço y Termas da Terronha Vimioso, situados en el Norte de Portugal.

Finalmente, en lo referente a esta categoría, se remarcan las ventajas y desventajas señaladas por los responsables de los establecimientos sobre las redes sociales. Entre los balnearios de Galicia la ventaja más repetida es la oportunidad de contacto con muchas personas de forma económica y rápida, mientras para el Norte de Portugal las ventajas indicadas están encaminadas a mejorar la marca de la empresa, ser un canal de ventas y un medio para comunicarse con los clientes. Como desventajas que encuentran en las redes sociales, los representantes de los balnearios de Galicia coinciden en que los comentarios negativos susceptibles de ser realizados acerca del establecimiento son la principal desventaja de este tipo de medios. En el caso portugués recalcan también los malos comentarios y se añade la falta de control, de filtraje, del material publicado. Por ello, se puede afirmar la preocupación de los empresarios respecto a los comentarios hechos por los usuarios, los cuales pueden perjudicar a la empresa, sobre todo a aquellas de dudosa credibilidad.

Se subrayan finalmente varias apreciaciones comentadas por los entrevistados. Respecto a los aspectos negativos que presentan las redes sociales, el Hotel Balneario Isla de la Toja, estima interesante plantear 
algún tipo de filtro, imparcial, que ayudase a detectar cuáles son comentarios reales y cuáles falsos. Por su parte, el Hotel Balneario de Guitiriz \& Golf Club y el Hotel Balneario Isla de la Toja significan la existencia de cierta coacción de los clientes respecto a hacer malos comentarios en las redes sociales, en caso de no recibir algún tipo de gratuidad en los servicios, hecho que debería ser corregido para evitar las críticas infundadas y la pérdida de credibilidad de los comentarios.

Finalmente, Termas de Arêgos, en el Norte de Portugal, plantea que sería productivo elaborar un grupo definido de personas interesadas únicamente en termalismo, con el fin de encaminar sus ofertas e información a este tipo de público concreto.

\section{¿Qué persiguen en las redes sociales?}

En función de las respuestas recibidas por los responsables de los balnearios de Galicia, se comprueba que los objetivos que persiguen en social media son variados, aunque la mayoría guarda nexos en común como: darse a conocer, captar y comunicarse con los clientes o servir como complemento a la promoción tradicional de forma económica y rápida.

Los establecimientos del Norte de Portugal, en general, persiguen objetivos diferentes, donde destaca promocionar el establecimiento, lanzar ofertas, captar clientes, darse a conocer y llegar a un número amplio de personas. Además, entre el colectivo portugués, uno de los propósitos es atraer público joven a los establecimientos termales, intentando romper con la percepción de que el balneario está ligado únicamente a personas mayores, curación de enfermedades, etc.; es decir, la vertiente médica del Turismo de Salud. El hecho de que las redes sociales sean utilizadas por un número amplio de gente joven interesa a los responsables de los balnearios, y ven en este medio una oportunidad de negocio. En la misma línea, otro objetivo muy comentado en las entrevistas de la región portuguesa, se encuentra: dar a conocer la prevención de ciertas dolencias con prácticas termales, sobre todo para niños/as y gente joven.

Por último, es preciso incidir en el objetivo al que alude Vidago Palace, pues es el único balneario de Galicia y del Norte de Portugal que indica tener entre sus objetivos canalizar clientes desde sus páginas de redes sociales a la página web, una estrategia muy acertada en materia de promoción online que destacan distintos profesionales en la materia, como Leung y Bai (2013), los cuales defienden la inclusión del link a la página oficial del establecimiento dentro de los post hechos en Facebook o Twitter, puesto que ayuda a enviar tráfico de las redes sociales a la web del establecimiento.

En cuanto al perfil de cliente que buscan captar en los medios sociales se puede concluir que, en general, los balnearios tienen como perfil potencial a personas de entre 15 y 55 años de edad, según los responsables de varios establecimientos aquellos son quienes más emplean las redes sociales, al igual que lo afirma Clarke, Montesinos, Montanera, y Bermúdez (2015) ${ }^{11}$. No especificándose en sus contestaciones otro tipo de características como pueden ser la procedencia, nivel de estudios, nivel de ingresos, etc.

\footnotetext{
${ }^{11}$ https://goo.gl/C96WNG
} 


\section{Actividad en las redes sociales}

La percepción de los responsables de los establecimientos sobre distintas cuestiones relacionadas con la actividad en las redes sociales permite explicar los siguientes resultados.

A nivel general, los comentarios que reciben los balnearios, tanto de Galicia como del Norte de Portugal, por parte de los usuarios de sus comunidades online, son por el momento, escasos. No obstante, al margen de su poca actividad, los comentarios recibidos, en su gran mayoría, son positivos y recalcan las cualidades del establecimiento o hablan de las experiencias vividas en el mismo. También son habituales las peticiones de información sobre tratamientos o condiciones del establecimiento, tanto por mensajes privados como por comentarios públicos. Destacan la existencia de comentarios que les ayudan a mejorar ciertos aspectos de la empresa, así como la motivación extra, en el caso de ser positivos, para realizar el trabajo del día a día. Ahora bien, se puede apuntar que dos balnearios de Galicia, concretamente el Hotel Balneario de Carballo y el Hotel Balneario Baños da Brea declaran que las encuestas de satisfacción son la herramienta más utilizada para percibir la opinión de sus clientes. Esto ocurre también en Termas das Caldas de Aregos, Termas da Moimenta y Termas das Taipas en el Norte de Portugal, lo que hace indicar que los responsables de los establecimientos termales siguen teniendo muy en cuenta las herramientas tradicionales para conocer la percepción de sus clientes.

En lo referente al tipo de contenido considerado como más apropiado por los responsables de los balnearios, se detecta que para los establecimiento de la eurorregión la actualización de su canal debe de incluir información de temática diferente, abarcando temas que estén relacionados con el propio establecimiento, ofertas e información de la zona en la cual se ubica el balneario, y noticias sobre termalismo y bienestar. Es esta una opción acertada si se tiene en cuenta que los manuales sobre gestión de perfiles y páginas de redes sociales recuerdan la importancia de publicar contenido variado, de tipo atractivo para el usuario, dirigido a provocar su participación y fidelización (Kim, Kim, y Wise, 2014). También han señalado lo conveniente y apropiado de publicar ofertas con el propósito de llegar al mayor número de clientes posible, aunque ciertamente, algunos de los balnearios defienden no abusar de este tipo de información, pues puede provocar una pérdida de interés por parte del usuario de los medios sociales.

Relacionado con la opinión de los responsables sobre la figura del Community Manager, es decir, la persona encargada de gestionar las redes sociales en la empresa, se inquiere que está incluida principalmente, en los establecimientos gallegos, en dos tipos de departamentos: El Departamento Comercial y de Marketing y el Departamento de Recepción. Además, existen dos balnearios cuya actividad en los medios sociales es gestionada por una empresa externa y un último establecimiento termal cuyo propietario es el encargado de esta tarea. En cuanto al departamento donde se localiza esta figura en los balnearios portugueses, se puede indicar que la gran mayoría de ellos, concretamente 7 de los entrevistados, sitúan a esta persona en el Departamento de Comunicación y Marketing, 2 en el Departamento de Recepción y un último balneario donde es el propietario es quien se encarga de este trabajo. Como caso curioso se detecta que 2 de los balnearios gallegos entrevistados, concretamente Hotel Balneario de Guitiriz \& Golf Club y Hotel Balneario de Compostela, apuntan que, dentro del Departamento de Recepción, el turno de noche, al tener menos carga de trabajo, es el más apropiado para dedicarse a la tarea de Community Manager. En cuanto a la planificación de este trabajador/a en los balnearios de la eurorregión, existen establecimientos que tienen 
estructurado y planificado este trabajo y otros en los cuales esta tarea es improvisada según se disponga de tiempo, una vez finalizado el trabajo principal del hotel-balneario.

En general, la mayoría de ellos considera que es un trabajo necesario debido a la influencia actual de los medios sociales para las empresas; sin embargo, en muchos casos, la persona encargada de las redes sociales ha de dedicarse además a otras tareas y compaginar ambos trabajos quedando relegado en muchas ocasiones la atención de este medio, a un segundo plano. Igualmente se detecta que una gran parte de los balnearios coincide en añadir que, por el momento, no puede contratar a una persona para dedicarla únicamente a esta labor. Vistas estas apreciaciones parece que la importancia destacada en un primer momento sobre el Community Manager, queda en entredicho.

Una vez efectuado el pertinente análisis de las entrevistas, se comprueba la existencia de unos medios sociales más valorados que otros. En este sentido, los balnearios de Galicia y Norte de Portugal eligen Facebook como red social principal para promocionarse. Las explicaciones aportadas por los responsables de los establecimientos de ambas regiones sobre la elección de Facebook, son variadas, aunque prima la explicación de que es la red social con más usuarios, lo cual les permite llegar a más personas, sobre todo público joven, pues en muchas ocasiones es el target que quieren atraer a sus balnearios. Asimismo, han de destacarse algunas respuestas de balnearios que, tanto gallegos como portugueses indican que lo ideal sería combinar la promoción entre varios tipos de medios sociales, tanto generalistas como especializados entre los balnearios que disponen de alojamiento, cual es el caso del Gran Hotel Isla La Toja en Galicia y Pedras Salgadas Spa \& Nature Park en el Norte de Portugal, quienes opinan que Facebook está más destinada a informar a los clientes y se puede complementar con otros medios como Booking o TripAdvisor, cuyo propósito está dirigido a la venta de un servicio.

\section{Percepción de rentabilidad de las redes sociales}

La rentabilidad de los medios sociales siempre ha sido un tema discutido, que ha acaparado la atención de los investigadores, para los cuales el ROI (Return On Investment) en las redes sociales ha sido una cuestión difícil de calcular (Cavalganti y Sobejano, 2011). Sin embargo, Rivero y Rangel (2016) demuestran, aunque de forma débil, que el disponer de un perfil en Facebook influye de forma positiva en mejorar la ocupación, por lo cual las empresas han de plantearse estar presentes en estos medios. Esta dificultad es destacada también por varios establecimientos termales de Galicia y del Norte de Portugal, pues ante la pregunta de la rentabilidad de los medios sociales dicen no saber con exactitud si este canal les genera o no algún tipo de rentabilidad.

En general, existen 6 balnearios que sí estiman rentables a las redes sociales y ven en éstas una ayuda para abaratar costes en materia de promoción; en cambio el resto de establecimientos entrevistados, coinciden en decir que, por el momento, no es un medio rentable, aunque aporten visibilidad y repercusión sobre la marca. 


\section{Autoevaluación y previsión futura}

Por último, se hace referencia a la autoevaluación y a la presión futura de las personas entrevistadas sobre las redes sociales. Así, se pueden dividir la información recabada entre aquellos balnearios gallegos y del Norte de Portugal que estiman llevar a cabo una gestión adecuada en los medios sociales, y quienes entienden que su actividad en este medio debería de mejorar, siendo mayor el número de establecimientos que comparten esta última consideración. Este hecho hace entrever la existencia de un elevado número de balnearios que perciben que su actividad en estos medios necesitaría ser más eficiente, dado que prácticamente la totalidad de los establecimientos, a excepción de Termas da Saúde en el Norte de Portugal, consideran que será un tipo de canal que continuará en el tiempo y evolucionará de forma distinta, con lo cual, prestarle atención en el presente es fundamental para su desarrollo de forma sostenible en el futuro.

\section{Conclusiones}

La investigación aquí desarrollada nos aproxima a la percepción de los responsables del Turismo Termal de la eurorregión Galicia-Norte de Portugal sobre uno de los medios más novedosos y actuales en materia de promoción turística, las redes sociales.

Los resultados alcanzados permiten dividir la opinión de las personas entrevistadas en dos grandes bloques de información, una positiva y otra negativa, generando una contradicción en las respuestas de los entrevistados.

Figura 1: Consideraciones generales de los balnearios de la Eurorregión sobre las redes sociales

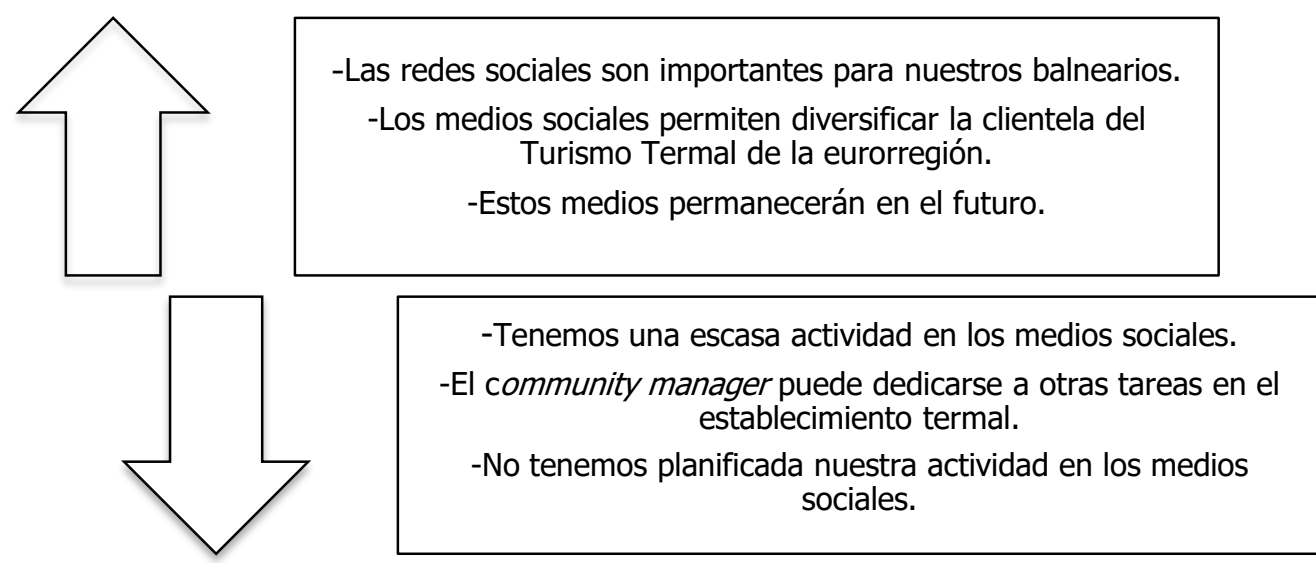

Fuente: Elaboración propia

A pesar de construirse estas opiniones discordantes, los resultados posibilitan describir la percepción, para el año 2015, de los responsables del termalismo de la eurorregión en el canal online.

De este modo, puede afirmarse en primer lugar que, a nivel general, sin profundizar en los aspectos individuales de cada balneario, existe cierta similitud entre los datos de los dos casos analizados (balnearios de Galicia y balnearios del Norte de Portugal). Así, en su gran mayoría, las personas entrevistadas exponen 
que las redes sociales son importantes para la promoción termal online de sus establecimientos, al ser éste un medio que permite contactar y comunicarse con un número elevado de personas, de forma fácil, rápida y a un coste bajo; siendo además un medio que permanecerá en el futuro.

Sin embargo, por otro lado, se detecta, un claro desinterés por este tipo de medios, al comentar que, por el momento, su actividad en las redes sociales es escasa, que el community manager debe priorizar en otras tareas antes que en la gestión de los medios sociales, o que no tienen planificada su actividad en este medio. Estos resultados resultan significativos para ampliar la investigación en este campo, mediante la posibilidad de combinar los datos con otro tipo de investigación de carácter cuantitativo, como pueden ser el estudio pormenorizado de los indicadores de cada página de redes sociales de los balnearios, con el propósito de comparar su situación real en el canal online.

\section{Mención de reconocimiento}

Una parte de los resultados de este artículo forman parte de las actividades de investigación promovidas a través de la Red XESCOM (R2014/026 XESCOM), apoyada por la Consellería de Cultura, Educación e Ordenación Universitaria de la Xunta de Galicia; de las tareas exploratorias del proyecto del Programa estatal de Fomento de la Investigación Científica y Técnica de Excelencia, Subprograma estatal de Generación de Conocimiento del Ministerio de Economía y Competitividad de España sobre Indicadores de gobernanza, financiación, rendición de cuentas, innovación, calidad y servicio público de las RTV europeas aplicables a España en el contexto digital (referencia CSO2015-66543-P); así como del Programa Prometeo de la

Secretaría Nacional de Educación Superior, Ciencia, Tecnología e Innovación (Senescyt) de Ecuador, desarrollado en la Universidad Técnica Particular de Loja (UTPL)

\section{Bibliografía}

Amaral, I. (2008). A @migração para o Ciberespaço: a Dimensão Social dos Mundos Virtuais. Observatorio $\left(O B S^{*}\right), 2(2), 325-344$

Bell, J. (2004). Como realizar um projecto de investigação. Lisboa: Gradiva.

Bonfada, M., Bonfada, P., Gândara, J., \& Brea, J. (2008). Turismo termal: Cambios conceptuales y mercadológicos de los balnearios en España. Turismo-Visão e Ação, 10(3), 415-434.

Booth, C. (2014). Experiences and wisdom behind the numbers: qualitative analysis of the National Action Alliance for Suicide Prevention's Research Prioritization Task Force stakeholder survey, American Journal of Preventive Medicine, 47(3), 106-114.

Casal, R. \& González, S. (2010). Os Balnearios de Galicia. Orixe e desenvolvemento. Santiago de Compostela: Servizo de Publicacións e Intercambio Científico Campus Vida.

Casaló, L. V., Flavián, C., \& Guinaliu, M. (2009). Importancia de las redes sociales en el turismo rural. Ponencia presentada en el II Congreso Internacional de Turismo Sostenible en Montaña, Huesca, España, 17-18 septiembre 2009

Castro, E. (2010). Estudio de casos como metodología de investigación y su importancia en la dirección y administración de empresas, Revista Nacional de Administración, 1(2), pp. 31-54. 
Cavalganti, J., \& Sobejano, J. (2011). Social Media IOR las relaciones como moneda de rentabilidad. Madrid: Bubuk Publishing

Celeya, J. (2008). La Empresa En La Web 2.0. Barcelona: Gestión 2000.

Chetty, S. (1996). The case study method for research in small and medium sized firms, International Small Business Journal, 15(1), 73-85.

Christakis, N \& Flowler, J. (2010). Conectados. El sorprendente poder de las Redes Sociales y cómo nos afectan. Madrid: Taurus.

Coller, X. (2000). Estudio de casos. Madrid, España: Centro de Investigaciones Sociológicas.

Coryn, C., Schröter, D., \& Mccowen, R. (2014). A Mixed Methods Study of Some of the Factors Associated With Successful School Strategies for Native Hawaiian Students in the State of Hawai, Journal of Mixed Methods Research, 8(4), 377-395.

Cruz, G. (2005). Promoción de destinos turísticos de la web: estrategias e indicadores para los destinos turísticos brasileños, Tesis Doctoral. Las Palmas de Gran Canaria: Universidad de Las Palmas de Gran Canaria.

De la Cruz Pulido, M \& Pulido, J. (2014). ¿Existe gobernanza en la actual gestión de los destinos turísticos?, Estudio de casos, PASOS. Revista de Turismo y Patrimonio Cultural, 12(4), 686-705.

Ellison, N., Steinfield, C., \& Lampe, C. (2007). The benefits of Facebook "friends:" Social capital and college students' use of online social network sites, Journal of Computer-Mediated Communication, 12(4), 1143-1168.

Fernández, L. (2006). ¿Cómo analizar datos cualitativos. Butlletí LaRecerca, 6, 1-13.

Galán, C., Edwards, P., Nelson, J., \& Van Der Wal, R. (2015). Digital innovation through partnership between nature conservation organisations and academia: A qualitative impact assessment, Ambio, 44(4), 538-549.

García, J. (2010). Comportamientos activos en usuarios 2.0: Facebook supera a Tuenti, la red social que había sido líder en España. Causas, estrategias de comunicación e impacto en la recepción. Observatorio $\left(O B S^{*}\right), 2(4), 153-166$.

Gonçalves, J., Fraiz, J., \& Manosso, F. (2013). Calidad de la experiencia en los hoteles termales de Galicia, España. Un análisis a través de la reputación online, Estudios y perspectivas en turismo, 22(3), 492-525.

Goodrich, J., \& Goodrich, G. (1987). Health care tourism. An exploratory study, Tourism Management, 8(3), 217-222.

Hernández, E., Fuentes, M., \& Morini, S. (2012). Una aproximación a la Reputación en Línea de los Establecimientos Hoteleros Españoles. Papers de Turisme, 52, 63-88.

Hoz, A. (2013). Entendiendo el turismo de salud: un análisis sociodemográfico, Escenarios: Empresa y Territorio, 2(2), 37-54.

Hudson, S., \& Thal, K. (2013). The Impact of Social Media on the Consumer Decision Process: Implications for Tourism Marketing, Journal of Travel \& Tourism Marketing, 30(1-2), 156-160.

Hyde, K. (2007). Contemporary information search strategies of destination-naïve international vacationers, Journal of Travel and Tourism Marketing, 21(2), 63-76.

Kang, M. y Schuett, M. (2013). Determinants of Sharing Travel Experiences in Social Media, Journal of Travel \& Tourism Marketing, 30(1-2), 93-107. 
Kaplan, M., \& Haenlein M. (2010). Users of the world, unite! The challenges and opportunities of Social Media, Business Horizons, 53(1), 59-68.

Kim, S., Kim, D., \& Wise, K. (2014). The effect of searching and surfing on recognition of destination images on Facebook pages, Computers in Human Behavior, 30, 813-823.

Leung, X., \& Bai, B. (2013). How Motivation, Opportunity, and Ability Impact Travelers' Social Media Involvement and Revisit Intention, Journal of Travel \& Tourism Marketing, 30(1-2), 58-77.

Martínez, A. (2011a). Turismo 2.0. Iniciando el despegue. Oviedo, España: Septem ediciones.

Martínez, L. (2012). Estrategias de promoción turística a través de Facebook, Palabra Clave, 15(2), 318-338.

Martínez, P. (2011b). El método de estudio de caso Estrategia metodológica de la investigación científica. Revista científica Pensamiento y Gestión, 20, 165-193.

Miles, M., Huberman, M., \& Saldaña, J. (2013). Qualitative Data Analysis. A methods Sourcebook. Arizona, USA: Sage.

Munar, A., \& Steen, J. (2014). Motivations for sharing tourism experiences through social media, Tourism Managment, 43, 46-54.

Pan, B., Maclaurin, T., \& Crotts, J. (2007). Travel blogs and their implications for destination marketing, Journal of Travel Research, 46(1), 35-45.

Patton, M. (2002). Qualitative research \& evaluation methods. Thousand Oaks, CA: Sage.

Rivero, M. S., \& Rangel, C. R. (2016). La influencia de la presencia en redes sociales sobre el grado de ocupación de los establecimientos turísticos. Revista de análisis turístico, (21), 1-10.

Scott, D. (2010). The new rules of marketing and PR. New Jersey, USA: John Wiley \& Sons, Inc.

VillareaL, O., \& Landeta, J. (2007). El estudio de Casos como metodología de investigación científica en Economía de la Empresa y dirección Estratégica. Investigaciones europeas de dirección y economía de la empresa, 16 (3), 31-52.

Yin, R. (1993). Applications of Case Study Research, Applied Social Research Methods Series. Newbury Park, CA: Sage Publications.

Yin, R. (1994). Case Study Research: Design and Methods. Thousand Oaks, CA: Sage Publications.

Zins, A. (2009). Deconstructing travel decision making and information search activities, Information and Communication Technologies in Tourism, 467-479. 